\title{
Bereits subklinische psychische Belastung erhöht das Risiko frühzeitiger Sterblichkeit
}

Fragestellung: Wie beeinflussen verschiedene Ausprägungen psychischer Belastung das Risiko frühzeitiger Sterblichkeit?

Hintergrund: Forschungsergebnisse der letzten Jahre deuten auf einen Zusammenhang zwischen psychischer Belastung und erhöhtem Risiko frühzeitiger Sterblichkeit hin. Allerdings mangelt es bislang an einer reliablen Schätzung des Risikofaktors und einer genauen Untersuchung des Kausalzusammenhangs.

Patienten und Methodik: Im Rahmen des Health Survey for England wurden von 1994 bis 2004 elf unabhängige Studien mit identischem Design in Privathaushalten durchgeführt. Grundlage für die vorliegende Metaanalyse bildet der Rohdatensatz der 68.222 Teilnehmer, die bei Baseline älter als 35 Jahre waren und keine relevante somatische Vorerkrankung aufwiesen.

Die psychische Belastung wurde mithilfe der 12-Item-Version des General Health Questionnaire (GHQ) erfasst, bei dem ab einem Gesamtwert von 4 von einer klinisch bedeutsamen Belastung auszugehen ist. Die Autoren differenzierten psychische Belastung in vier Ausprägungsgrade $(0=$ asymptomatisch, $1-3=$ subklinisch symptomatisch, $4-6=$ symptomatisch, $7-12=$ hoch symptomatisch). Die aus den Sterbeurkunden gewonnenen Todesursachen wurden anhand von ICD-9 und -10 klassifiziert. Zur Untersuchung einer inversen Kausalität schloss man alle Probanden aus der Analyse aus, die in den ersten fünf Follow-up-

Jahren verstorben waren.
Russ T, Stamatakis E, Hamer M et al. Association between psychological distress and mortality: individual participant pooled analysis of 10 prospective cohort studies. BMJ 2012; 345: e4933
Ergebnisse: Auf Basis der 8.365 Todesfälle im Followup-Zeitraum (im Mittel 8,3 Jahre, SD: 3,5) konnte ein signifikanter Zusammenhang zwischen psychischer Belas-

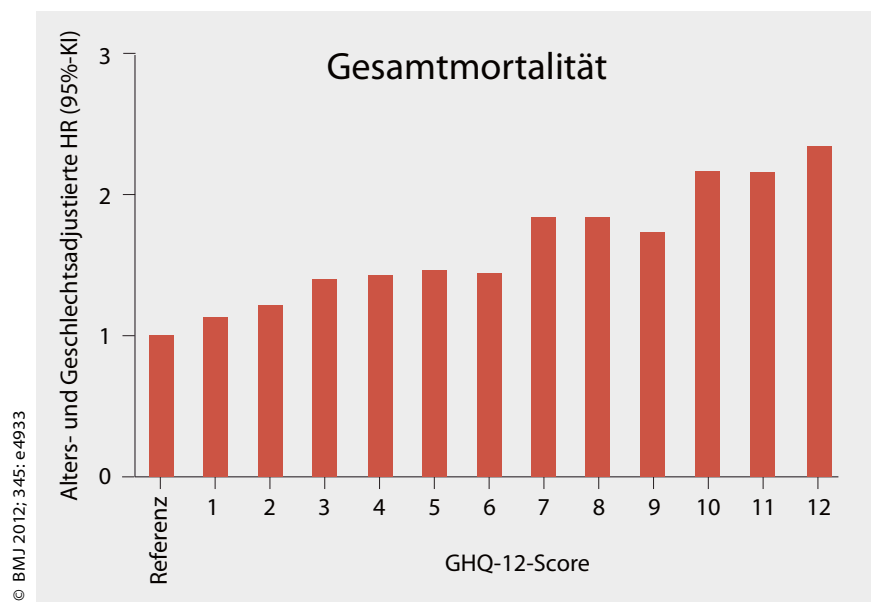

1 Die Sterblichkeit steigt mit der psychischen Belastung. tung bei Baseline und Sterbewahrscheinlichkeit gezeigt werden - unabhängig von der Schwere der Belastung und der Todesursache $(\mathrm{p}<0,001)$. Im Sinne einer Dosis-Wirkungs-Beziehung fiel das Sterberisiko pro Standardabweichung höherer Belastung im GHQ um 1,21 ungünstiger aus ( $\bullet$ Abb. 1). Differenzierte man nach Todesursachen, hatten schon subklinisch belastete Teilnehmer ein um 29\% erhöhtes Risiko, aufgrund einer kardiovaskulären Erkrankung oder durch eine externe Ursache zu versterben (pro SD im GHQ um 1,22 und 1,26 ungünstigerer Ratio). Im Gegensatz dazu stieg die Wahrscheinlichkeit, an einer Krebserkrankung zu versterben, erst ab einer hohen psychischen Belastung (GHQ-Score 7-12) signifikant auf $41 \%$ und dann je SD im GHQ um weitere $9 \%$ an.

Schlussfolgerungen: Die Ergebnisse sprechen für einen proportionalen Anstieg des Sterberisikos mit dem Ausmaß psychischer Belastung - auch schon bei subklinischen Symptomen und unabhängig von der Todesursache. Lediglich in Bezug auf Krebserkrankungen scheint sich psychische Belastung erst ab höheren Belastungslevels auf das Sterberisiko auszuwirken.

\section{- Kommentar von Valeska Pape \\ Das Risiko steigt mit der Belastung}

Bei der vorgestellten Studie handelt es sich um die erste einzelfallbasierte Metaanalyse, die auf Grundlage einer großen bevölkerungsbasierten Stichprobe einen Zusammenhang zwischen psychischer Belastung und Sterberisiko zeigen konnte. Die genaue statistische Analyse unter Berücksichtigung zahlreicher relevanter Kontextvariablen erlaubt die Annahme, dass sich auch schon subklinisch ausgeprägte psychische Symptome kausal auf die Mortalität auswirken und dieser Effekt mit der Schwere der Belastung wächst. Lohnend für zukünftige Studien scheint die Frage, aus welchen direkten (z.B. physiologischen) und indirekten (z. B. verhaltensmäßigen) Prozessen sich dieser zusammensetzt - und inwieweit er durch verschiedene Behandlungsansätze zu beeinflussen ist.

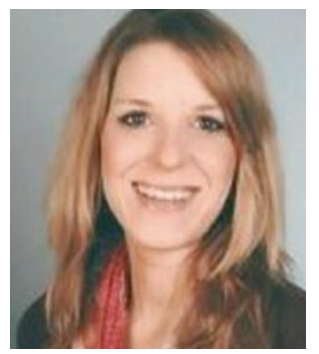

Dipl.-Psych. Valeska Pape (PPiA)

Klinik für Psychiatrie und Psychotherapie der Universitätsmedizin Mainz E-Mail:

valeska.pape@unimedizin-mainz.de 